\title{
Trajectory of Lung Function Change in Childhood Cancer Survivors: An Exploratory Analysis
}

\author{
Katina Zheng ${ }^{1}$, Mylene Bassal ${ }^{2}$, Jemila Hamid ${ }^{3}$, Franco Momoli², Joe Reisman², Vimoj \\ Nair $^{3}$, and Dhenuka Radhakrishnan ${ }^{4}$ \\ ${ }^{1}$ University of Ottawa Faculty of Medicine \\ ${ }^{2} \mathrm{CHEO}$ \\ ${ }^{3} \mathrm{CHEO}$ Research Institute \\ ${ }^{4}$ Children's Hospital of Eastern Ontario
}

May 28, 2020

\begin{abstract}
Introduction Although five-year survival rates for childhood cancer have surpassed $85 \%$, childhood cancer survivors continue to suffer from long-term effects decades after treatment completion. The prevalence of pulmonary dysfunction is very common at $65.2 \%$ in adulthood. This study explores early changes in the trajectory of pulmonary function in pediatric cancer patients who received pulmonary toxic therapy. Methods In this single-center, retrospective cohort study, we included pediatric cancer patients diagnosed at $<18$ years old between January 1994 and December 2014. Patients were included if they received pulmonary toxic exposure: either chemotherapy (bleomycin, busulfan, lomustine carmustine or cyclophosphamide) or thoracic radiation. Outcomes included percent predicted values for spirometry, lung volumes, and diffusion capacity of the lungs post-treatment. Results Of 86 children who met inclusion criteria, 99\% received pulmonary toxic chemotherapy, and $79 \%$ received thoracic radiotherapy. Patients showed an overall decrease in all three lung function parameters immediately posttreatment. Between one- and four-years post-treatment, there was a larger sustained decline in percent predicted lung function parameters for females (mean Forced Expiratory Volume in 1 Second, FEV1=81.0\% \pm 15.7$)$ than males $(\mathrm{FEV} 1=93.2 \% \pm 10.2)$. Sensitivity analysis of 65 children who received radiation and bleomycin revealed pulmonary function trends similar to the overall population. Conclusions Our results reveal that male and female patients experience different lung function trajectories following pulmonary toxic cancer treatment, with females performing more poorly over time despite similar baseline function. Further research is needed to better understand the factors associated with poor lung function and impaired recovery post-treatment, particularly in females.
\end{abstract}

\section{Trajectory of Lung Function Change in Childhood Cancer Survivors: An Exploratory Analysis}

Katina Zheng, BHSc ${ }^{1}$; Mylène Bassal, MDCM ${ }^{1,2,3}$; Jemila Seid Hamid, PhD $^{3}$, Franco Momoli, PhD ${ }^{3,6}$ Joe $^{2}$ Reisman, $\mathrm{MD}^{1,3,7}$; Vimoj Nair, $\mathrm{MD}^{3,5,6}$, Dhenuka Radhakrishnan, $\mathrm{MD}^{1,3,4,7}$

1 Faculty of Medicine, University of Ottawa

${ }^{2}$ Department of Pediatrics, Division of Hematology/Oncology, Children's Hospital of Eastern Ontario

3 Children's Hospital of Eastern Ontario Research Institute

${ }^{4}$ ICES uOttawa, Ottawa, Ontario

5 Division of Radiation Oncology, University of Ottawa

${ }^{6}$ Ottawa Hospital Research Institute, Ottawa, Canada 
${ }^{7}$ Department of Pediatrics, Division of Respirology, Children's Hospital of Eastern Ontario

This work was performed at The Children's Hospital of Eastern Ontario (CHEO), ON, Canada.

Corresponding author information: Dr. Dhenuka Radhakrishnan, CHEO, 401 Smyth Rd, Ottawa, ON K1H 8L1. Email: dradhakrishnan@cheo.on.ca. Phone number: 613-737-7600

Address requests for reprints to: Dr. Dhenuka Radhakrishnan, CHEO, 401 Smyth Rd, Ottawa, ON K1H 8L1. Email: dradhakrishnan@cheo.on.ca. Fax: 613-738-4297. Telephone:613-737-7600

Conflicts of Interest and Source of Funding: The authors have no disclosures regarding conflicts of interest. This study was funded by the Children's Hospital of Eastern Ontario Research Institute.

Abbreviated Title: Trajectory of Lung Function Post-Cancer Treatment

\section{Key Words: late effects, pulmonary function, chemotherapy, radia- tion therapy, cancer, children}

Main Text Word Count: 3066

Abstract

Introduction

Although five-year survival rates for childhood cancer have surpassed 85\%, childhood cancer survivors continue to suffer from long-term effects decades after treatment completion. The prevalence of pulmonary dysfunction is very common at $65.2 \%$ in adulthood. This study explores early changes in the trajectory of pulmonary function in pediatric cancer patients who received pulmonary toxic therapy.

\section{Methods}

In this single-center, retrospective cohort study, we included pediatric cancer patients diagnosed at $<18$ years old between January 1994 and December 2014. Patients were included if they received pulmonary toxic exposure: either chemotherapy (bleomycin, busulfan, lomustine carmustine or cyclophosphamide) or thoracic radiation. Outcomes included percent predicted values for spirometry, lung volumes, and diffusion capacity of the lungs post-treatment.

\section{Results}

Of 86 children who met inclusion criteria, $99 \%$ received pulmonary toxic chemotherapy, and $79 \%$ received thoracic radiotherapy. Patients showed an overall decrease in all three lung function parameters immediately post-treatment. Between one- and four-years post-treatment, there was a larger sustained decline in percent predicted lung function parameters for females (mean Forced Expiratory Volume in 1 Second, FEV1=81.0\% $\pm 15.7)$ than males $(\mathrm{FEV} 1=93.2 \% \pm 10.2)$. Sensitivity analysis of 65 children who received radiation and bleomycin revealed pulmonary function trends similar to the overall population.

\section{Conclusions}

Our results reveal that male and female patients experience different lung function trajectories following pulmonary toxic cancer treatment, with females performing more poorly over time despite similar baseline function. Further research is needed to better understand the factors associated with poor lung function and impaired recovery post-treatment, particularly in females. [248 words]

\section{Introduction}

Over the last few decades, improved antineoplastic therapies have resulted in significant improvements in cancer survival rates. For childhood and adolescent cancers, the 5-year survival has risen to over $85 \%^{1}$, 
creating a growing population of childhood cancer survivors (CCS). In 2015, over 360,000 CCS were living in the United States ${ }^{1}$.

Although treatment regimens, which can include radiation, chemotherapy, and surgery, are highly effective, they can also cause significant long-term morbidity and mortality as these young survivors age. Secondary malignancies and organ dysfunction can develop just a few years after the successful cure of the primary cancer $^{2}$. By thirty years post- diagnosis, over $70 \%$ of CCS will suffer from a chronic health condition ${ }^{3}$.

While pulmonary dysfunction is the second leading cause of mortality in $\mathrm{CCS}^{4}$, it is also one of the most common morbidities of treatment, with a prevalence of $65.2 \%$ in adulthood ${ }^{5}$. The increase in prevalence of pulmonary morbidity over time may be due to lung injury occurring during a period of lung development, leaving a deficit in growth potential, thereby amplifying the natural decline in lung function with age ${ }^{6}$. Alternatively, lungs may be more susceptible to the toxic effects of radiation and immunosuppression during this growth phase leading to ongoing damage even after completion of treatment. Common pulmonary toxic therapies, including bleomycin, busulfan, lomustine, carmustine, and thoracic radiation have been shown to result in sequelae including pulmonary fibrosis, and interstitial pneumonitis ${ }^{7}$ which can result in progressive lung damage. Radiation can also cause hypoxia and oxidative stress, leading to long-term tissue damage, and chronic inflammation ${ }^{8,9}$.

Improved understanding of the early trajectory of pulmonary dysfunction in CCS can improve early detection and facilitate treatment to prevent or reduce associated morbidities, as well as improve decision-making surrounding the use and dosage of pulmonary toxic therapies in certain subpopulations during treatment.

In this retrospective cohort study, we sought to better understand the early trajectory of lung function and explore impacting factors in children who received pulmonary toxic cancer therapies. Through cross-sectional pediatric and adult studies, we know that lung function is altered in this population, but the onset and early trajectory of pulmonary function change is not well characterized.

\section{Methods}

\section{Study Design}

We performed a retrospective cohort study of children who received pulmonary toxic chemotherapy or thoracic radiation for treatment of childhood cancer over a twenty-year period and observed the trajectory of lung function up to four years after the completion of cancer treatment. This study was approved by the Research Ethics Board of the Children's Hospital of Eastern Ontario (CHEO) and is reported in accordance with the STROBE statement ${ }^{10}$.

\section{Participants and Setting}

We included all patients diagnosed and treated at CHEO between January 1994 and December 2014 for a diagnosis of Hodgkin Lymphoma (HL), Wilms Tumour (WT) or extracranial germ cell tumour (GCT). WT and GCT are two types of embryonal tumours. CHEO is a tertiary care pediatric hospital in Ottawa, Canada with a medium-sized pediatric oncology program, treating approximately 70 new oncology patients per year. Included patients were $<18$ years at diagnosis and received both pulmonary function testing and pulmonary toxic treatment. We limited inclusion to children with a minimum of two acceptable pulmonary function tests (PFT), with at least one test performed within 90 days following treatment completion and prior to any relapse, in order to capture the onset of pulmonary function changes related to initial treatment. Our centre only performs pulmonary function testing on children 6 and older, so children below this age cut-off were excluded.

\section{Pulmonary Function Evaluation Outcomes}

The main study outcome was percent predicted forced expiratory volume in one second $\left(\mathrm{FEV}_{1}\right)$ as measured using spirometry. Secondary outcomes were total lung capacity (TLC), measured using body plethysmography, and diffusion capacity of the lungs for carbon monoxide (DLCO), which was adjusted for the patient's hemoglobin level when a recent value was available $\left(\mathrm{DLCO}_{\mathrm{adj}}\right)$. Trained respiratory therapists performed 
and independently interpreted all PFTs for acceptability and reproducibility at the time of test completion in accordance with American Thoracic Society standards ${ }^{11}$. Pulmonary function was measured with the Vmax@ Encore PFT system(Care Fusion, Yorba Linda, CA), and a modified version of the Morris et al. reference equations was used to determine percent predicted values, which were adjusted for patient age, sex and height ${ }^{12}$. All available PFTs performed from 30 days pre-diagnosis were collected retrospectively from patients' medical records.

As per routine care practices at CHEO, all children who received pulmonary toxic therapy underwent pulmonary function testing at the end of treatment regardless of the presence of symptoms. For patients with one or more recurrences of their primary cancer, all treatment and pulmonary function data beyond the date of the relapse was censored and excluded from the analysis. This ensured that analysis was limited to lung function changes solely due to initial pulmonary toxic exposure and to avoid any confounding effects of cumulative treatment exposures due to relapse.

\section{Primary Exposure: Pulmonary Toxic Therapy}

Agents with significant pulmonary toxic properties identified from the literature include pulmonary radiation and select chemotherapy agents (bleomycin, busulfan, carmustine, lomustine and cyclophosphamide). While cyclophosphamide is not traditionally considered by oncologists to be a pulmonary toxic chemotherapy agent, it is well-reported to cause multiple lung sequelae including early and late onset pneumonitis and pulmonary fibrosis ${ }^{13}$. We stratified thoracic radiation into high volume or low volume depending on the volume of lung included in the radiation field. Mantle, mediastinum, whole lung and total body irradiation were considered high-volume thoracic radiation, while neck, abdominal, inverted Y, para-aortic, flank or supraclavicular radiation were considered low volume thoracic radiation. Internationally approved standardized pediatric oncology treatment protocols based on cancer type and extent of disease were used for both chemotherapy and radiation therapy. These were consistent throughout the study time period.

\section{Additional Variables}

Variables examined in this study also included patient demographics (age, height, weight, sex, past medical history), cancer characteristics (diagnosis, sites of disease, staging, relapse history), treatment history (chemotherapy agents, dates and dosage, radiation fields and dosage, bone marrow transplant or thoracic surgery). Side effects of therapy with the potential to affect measured pulmonary function such as pneumonitis and allergic reactions, and hospital inpatient admissions and emergency department visits of a respiratory cause (e.g. pneumonia, atelectasis) were also captured. Data were collected retrospectively from 30 days prior to patient diagnosis from medical records at CHEO, including local hospital health administrative data for health care visit dates.

\section{Statistical Analysis}

Descriptive statistics were summarized for the primary and secondary outcomes as well as demographics, therapeutic exposures, hospitalizations and other clinical variables. These summary measures were compared at baseline for the overall study cohort, at various time periods both before and after treatment completion, and for females and males separately. Mean and standard deviation (SD) or median and interquartile range (IQR) were used for continuous outcomes. Binary outcomes were summarized using frequency and percentages.

Given the retrospective nature of the study, lung function testing was performed at variable time points in coordination with treatments or follow up clinic visits. Therefore, data was summarized in monthly intervals, to allow comparability of the measurements across patients and to allow formal longitudinal investigation of the trajectories for the various outcomes considered. Hence, for each child, the mean outcome values across all visits within a given month were used as the monthly value of the outcome.

The visit date for the last recorded cancer treatment (radiation or chemotherapy) was defined as time zero. Data was summarized into intervals before and after treatment completion, with the post-treatment timeperiod further subdivided into within one year (immediate effect), between one year and four years (short 
term effect) and after four years (long term effect). These periods were defined after careful consideration of the distribution of the primary and secondary outcomes and the corresponding time trajectories.

Exploratory analyses were done to investigate lung function trajectories over time for all three pulmonary function outcomes (FEV1, TLC and $\mathrm{DLCO}_{\mathrm{adj}}$ ), and to evaluate similarities and differences in lung function trajectories before and after cancer treatment. Specifically, lung function trajectory was stratified by sex to determine if there were any differences between groups. To determine the potential additive pulmonary toxic effects, sensitivity analyses were performed to explore differences in lung function trajectories among a subgroup of children who received both chemotherapy and radiation therapy.

All analysis was performed using the $\mathrm{R}$ statistical software package ${ }^{14}$.

\section{Results}

Over the twenty-year study period from January 1994 to December 2014, 212 children were diagnosed with HL, GCT or WT at CHEO. Of these, 86 met inclusion criteria for both pulmonary toxic treatment exposure and pulmonary function testing (Figure 1).

Characteristics of included patients are presented in Table 1. The median age at diagnosis for included patients was $14.95[12.2,16.0]$ years. A significant proportion $(14.0 \%)$ reported respiratory symptoms at diagnosis, and $14.0 \%$ had evidence of malignancy in the lungs. In total, $22.1 \%$ had a complication that included either an emergency department visit or inpatient admission for a respiratory cause between their date of diagnosis and 1-year post-treatment completion. During follow-up, approximately $12.8 \%$ and $17.4 \%$ reported ever smoking tobacco or marijuana respectively, and $14.0 \%$ have had an asthma diagnosis. None of the patients in the study cohort received busulfan, lomustine, or thoracic surgery, which are common causes of pulmonary dysfunction ${ }^{15}$. Carmustine was excluded from analysis because it was only used post-relapse. Reactions linked to bleomycin were documented or suspected in four patients, and were considered mild (e.g., pneumonitis, rash). Thirteen $(15.1 \%)$ children relapsed following their initial treatment, after which their treatment and pulmonary function data were censored. Five (5.8\%) patients died before the end of the study period and were also censored at the date of death.

Given the difference in treatment protocols, patients with a diagnosis of HL had shorter treatment duration and larger doses of pulmonary toxic chemotherapy, on average, than patients with WT or GCT. The median time from treatment start to treatment completion was $4[3,6]$ months, and follow-up (time from diagnosis to last recorded pulmonary function test) was $19[13,31]$ months. Characteristics of the study population throughout and following treatment are shown in Table 2.

Overall, from pulmonary function testing, there were a total of $404 \mathrm{FEV}_{1}$ measurements, for an average of 4.7 per patient. There were $387 \mathrm{TLC}$ and $328 \mathrm{DLCO}_{\text {adj }}$ measurements. Analysis of lung function data revealed that a greater proportion of patients with lung function below $80 \%$ predicted were female than male. Further analysis stratified by sex revealed that females showed a different trajectory and had lower lung function than males post-treatment, despite similar lung function pre-treatment (Figure 2). During treatment, females

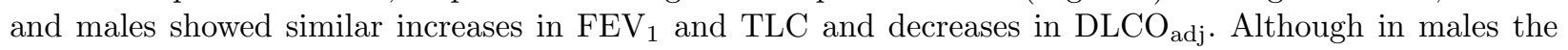
lung function recovered to baseline or improved post-treatment, females continued to decline and eventually stabilized at a lower percent predicted value. This divergence in lung function persisted throughout follow-up, to 80 months post-treatment. This was consistent across all three lung function outcomes $\left(\mathrm{FEV}_{1}, \mathrm{DLCO}_{\text {adj }}\right.$, and TLC). The breakdown of lung function outcomes during and post treatment are shown in Table 3.

Additional descriptive statistical analysis stratified by sex shows poorer lung function in females despite being exposed to fewer factors that could explain this difference. Females tended to be diagnosed at an older age and receive less radiation and pulmonary toxic chemotherapy than males (E-table 1 - Females and E-table 2 - Males).

Sensitivity analysis of children who received both radiation therapy (pooled high and low volume radiation therapy exposure) and bleomycin $(\mathrm{n}=65)$ showed similar lung function trajectories compared to the overall population (E-image 1). 


\section{Discussion}

This study is one of the first to explore the early trajectory of pediatric lung function following pulmonary toxic therapy using objective pulmonary function testing. Overall, we observed that children treated for childhood cancers with pulmonary toxic therapies experience three key phases in pulmonary function change, (1) a transient change in pulmonary function throughout treatment "immediate effect", (2) a sharp drop in pulmonary function immediately following treatment completion "short term effect", and (3) slow recovery in pulmonary function starting months after the end of treatment "long term effect".

We suspect that the increase of $\mathrm{FEV}_{1}$ and TLC in the immediate period resulted from a combination of cancer location and resolution of an acute illness. Mediastinal masses, which are commonly found in HL, can decrease pulmonary function and present with respiratory symptoms at diagnosis ${ }^{16}$. Patients presenting with respiratory symptoms have been seen to have an average decrease in $\mathrm{FEV}_{1}$ of up to $28 \%$, compared to asymptomatic patients ${ }^{16}$. Thus, tumour shrinkage with treatment may have played a role in the initial rise of lung function. Additionally, certain lung function tests like spirometry are effort-dependent and thus values may increase as patients experience improved health towards the end of their treatments, despite our inclusion of only acceptable and reproducible PFTs.

In the short term, we suspect that chest wall or more likely lung inflammation as in radiation pneumonitis may play a role in the acute but short-term drop in pulmonary function outcomes in the first few months posttreatment. Radiation pneumonitis is a well-documented side effect of treatment, often occurring within the first two to four months post-radiation ${ }^{17}$, and has been previously shown to cause decreases in both $\mathrm{FEV}_{1}$ and $\mathrm{DLCO}^{18}$. Acute drops in pulmonary function can also be seen with bleomycin-induced pneumonitis, which occurs in up to $46 \%$ of patients, and can decrease both TLC and DLCO ${ }^{19,20}$ though only a portion of our study population received this treatment.

In the long term, our results show that most children recover pulmonary function within a year following pulmonary toxic treatment. While this may be an observation secondary to overall improved health, energy and ability to provide a maximal effort during pulmonary function testing, interestingly, we found a difference in the degree of lung function recovery between males and females. Furthermore, DLCO is an effort-independent measure and also did not recover as quickly or fully in females as in males. Key factors such as age at diagnosis, treatment exposure did not explain the divergence in lung function over time. This suggests that sex may influence lung function following exposure to pulmonary toxic therapies resulting from cancer treatment. The adult literature suggests that women may be at greater risk of lung injury as a result of receiving similar radiation treatment protocols despite having smaller average lung volumes ${ }^{21}$. Another consideration is the hormonal effect of puberty on lung function. Previous reports have shown that lung function measured by plethysmography and single breath gas transfer (TLC and DLCO respectively) in healthy children rise discontinuously during puberty for males and correlate with an increase in thoracic volume, but this increase is not seen in females ${ }^{22}$. This sex difference has also been demonstrated during puberty when measuring $\mathrm{FEV}_{1}{ }^{23}$. This natural difference in lung growth may contribute to the difference in lung function recovery from pulmonary toxic treatment during this important phase of development. Additionally, females have been shown to experience disproportionate negative effects of cancer treatments on their lung function and mortality compared to their male counterparts ${ }^{2}$. Similar sex-related differences in pulmonary function and poorer health outcomes among females after puberty have been shown in other lung diseases including asthma and cystic fibrosis ${ }^{24-26}$.

The high rate of respiratory complications which included respiratory-related ED visits or admissions indicates that complications often happen early (Table 1). Additionally, although it is known that years following treatment, CCS suffer disproportionally from pulmonary issues such as restrictive, or obstructive disease, and diffusion defects compared to the general population ${ }^{27-29}$, our results also suggest there may also be a difference between male and female lung function trajectories that begins very early following treatment completion.

Despite smoking and marijuana use being reported in patient medical records at various time points during 
follow-up, because these habits are not routinely asked for in children, it was not possible to know whether this exposure was present at the time of onset of lung function changes. Nevertheless, we found smoking rates similar to the rest of Ontario's pediatric population, with previous studies reporting that $16 \%$ of Ontario $7^{\text {th }}$ to $12^{\text {th }}$ graders had smoked in the last year and $19 \%$ had used marijuana ${ }^{30}$. The prevalence of asthma in our cohort was below the provincial prevalence in youth, which can be up to $28 \%$ in males 10-14 years, and suggests that treating clinicians may under-recognize the prevalence of asthma among oncology patients ${ }^{31}$.

In our sensitivity analysis that compared lung function trajectory in patients who received radiation therapy in conjunction with bleomycin $(n=65)$, we found it did not differ significantly from the full cohort. Nevertheless, due to the limited sample size, and because radiation is a known potentiator of the pulmonary toxic effects of bleomycin therapy ${ }^{32-34}$ that correlates with greater rates of interstitial pneumonitis, fibrosis, and mortality, patients who receive this combined treatment should be carefully monitored through pulmonary function testing at regular intervals even in the absence of clinical symptoms. Despite the observational design, one important strength of our study lies in that all children had lung function testing at the end of their treatment, regardless of symptoms. This thus reduces the potential risk of bias caused by a disproportionately higher number of PFTs being performed in children with respiratory dysfunction.

One potential limitation of this study was our lack of a control group. However, as we used percent predicted lung function measures as our outcome, these are inherently referenced against a control population and are adjusted for height, age, and sex, thereby negating the need for a control group as in other longitudinal studies. Given our small sample size and single center design, we were limited in our ability to explore individual patient lung function trajectories and thus only powered to explore the overall trajectory of lung function in our cohort. The small sample size further limited our ability to explore a larger number of clinical predictors (e.g. age, treatment dosage, comorbidities) that could affect lung function trajectory beyond the effect of sex and the combination of bleomycin with radiation.

Our study sheds light on important factors to consider in follow-up of childhood cancer survivors, especially in females who may be more vulnerable to the pulmonary toxic effects of cancer treatment. This exploratory analysis of lung function trajectory in CCS provides several hypotheses upon which to design a future study looking at a longer period of follow up, or a prospective multicentre study to increase sample size, and better understand the onset and contributing factors that influence lung function outcomes in this vulnerable population.

\section{References}

1. Noone AM, Howlader N, Krapcho M, Miller D, Brest A, Yu M, Ruhl J, Tatalovich Z, Mariotto A, Lewis DR, Chen HS, Feuer EJ CK. Section 28 Childhood Cancer by Site Incidence, Survival and Mortality. 2015. http://www.ncbi.nlm.nih.gov/pubmed/28055103

2. Armstrong GT, Liu Q, Yasui Y, Neglia JP, Leisenring W, Robison LL, Mertens AC. Late mortality among 5-year survivors of childhood cancer: A summary from the childhood cancer survivor study. Journal of Clinical Oncology. 2009;27(14):2328-2338. doi:10.1200/JCO.2008.21.1425

3. Oeffinger KC, Mertens AC, Sklar CA, Kawashima T, Hudson MM, Meadows AT, Friedman DL, Marina N, Hobbie W, Kadan-Lottick NS, et al. Chronic Health Conditions in Adult Survivors of Childhood Cancer. New England Journal of Medicine. 2006;355(15):1572-1582. http://www.nejm.org/doi/abs/10.1056/NEJMsa060185. doi:10.1056/NEJMsa060185

4. Huang T-T, Hudson MM, Stokes DC, Krasin MJ, Spunt SL, Ness KK. Pulmonary Outcomes in Survivors of Childhood Cancer. Chest. 2011;140(4):881-901. http://linkinghub.elsevier.com/retrieve/pii/S0012369211605242. doi:10.1378/chest.10-2133

5. Hudson MM, Ness KK, Gurney JG, Mulrooney DA, Chemaitilly W, Krull KR, Green DM, Armstrong GT, Nottage KA, Jones KE, Sklar CA, Srivastava DK RL. Clinical Ascertainment of Health Outcomes among Adults Treated for Childhood Cancer: A Report from the St. Jude Lifetime Cohort Study. Jama. 2013;309(22):2371-2381. doi:10.1001/jama.2013.6296.Clinical 
6. Gower WA, Collaco JM, Mogayzel PJ. Lung Function and Late Pulmonary Complications Among Survivors of Hematopoietic Stem Cell Transplantation During Childhood. Paediatric Respiratory Reviews. 2010;11(2):115-122. http://dx.doi.org/10.1016/j.prrv.2010.01.006. doi:10.1016/j.prrv.2010.01.006

7. Children's Oncology Group. Long-Term Follow-Up Guidelines for Survivors of Childhood, Adolescent, and Young Adult Cancers Version 5.0. 2018.

8. Fleckenstein K, Zgonjanin L, Chen L, Rabbani Z, Jackson IL, Thrasher B, Kirkpatrick J, Foster WM, Vujaskovic Z. Temporal Onset of Hypoxia and Oxidative Stress After Pulmonary Irradiation. International Journal of Radiation Oncology Biology Physics. 2007;68(1):196-204. doi:10.1016/j.ijrobp.2006.12.056

9. Graves PR, Siddiqui F, Anscher MS, Movsas B. Radiation pulmonary toxicity: From mechanisms to management. Seminars in Radiation Oncology. 2010;20(3):201-207. http://dx.doi.org/10.1016/j.semradonc.2010.01.010. doi:10.1016/j.semradonc.2010.01.010

10. von Elm E, Altman DG, Egger M, Pocock SJ, Gøtzsche PC, Vandenbroucke JP. The strengthening the reporting of observational studies in epidemiology (STROBE) statement: Guidelines for reporting observational studies. International Journal of Surgery. 2014;12(12):1495-1499. doi:10.1016/j.ijsu.2014.07.013

11. Culver BH, Graham BL, Coates AL, Wanger J, Berry CE, Clarke PK, Hallstrand TS, Hankinson JL, Kaminsky DA, MacIntyre NR, et al. Recommendations for a Standardized Pulmonary Function Report. An Official American Thoracic Society Technical Statement. American Journal of Respiratory and Critical Care Medicine. 2017;196(11):1463-1472. http://www.atsjournals.org/doi/10.1164/rccm.201710-1981ST. doi:10.1164/rccm.201710-1981ST

12. Morris JF, Koski A, Johnson LC. Spirometric Standards for Healthy Nonsmoking Adults. Journal of Occupational and Environmental Medicine. 1971;103(1):360. doi:10.1097/00043764-197107000-00021

13. Malik SW, Myers J, DeRemee R, Specks U. Lung Toxicity Associated with cyclophosphamide use. Two distinct patterns. Am J Respir Crit Care Med. 1996;154(6). doi:10.1164/ajrccm.154.6.8970380

14. R Core Team. R: A language and environment for statistical computing. 2018. https://www.r-project.org

15. Huang T-T, Hudson MM, Stokes DC, Krasin MJ, Spunt SL, Ness KK. Pulmonary Outcomes in Survivors of Childhood Cancer. Chest. 2011;140(4):881-901. http://dx.doi.org/10.1378/chest.10-2133. doi:10.1378/chest.10-2133

16. King DR, Patrick LE, Ginn-Pease ME, McCoy KS, Klopfenstein K. Pulmonary function is compromised in children with mediastinal lymphoma. Journal of Pediatric Surgery. 1997;32(2):294-300. doi:10.1016/S00223468(97)90197-4

17. Versluys AB, Bresters D. Pulmonary Complications of Childhood Cancer Treatment. Paediatric Respiratory Reviews. 2016;17:63-70. http://dx.doi.org/10.1016/j.prrv.2015.09.004. doi:10.1016/j.prrv.2015.09.004

18. Miller KL, Zhou SM, Barrier RC, Shafman T, Folz RJ, Clough RW, Marks LB. Long-term changes in pulmonary function tests after definitive radiotherapy for lung cancer. International Journal of Radiation Oncology Biology Physics. 2003;56(3):611-615. doi:10.1016/S0360-3016(03)00182-2

19. Sleijfer S. Bleomycin-induced pneumonitis. Chest. 2001;120(2):617-624. doi:10.1378/chest.120.2.617

20. Wolkowicz J, Sturgeon J, Rawji M, Chan CK. Bleomycin-induced pulmonary function abnormalities. Chest. 1992;101(1):97-101. http://dx.doi.org/10.1378/chest.101.1.97. doi:10.1378/chest.101.1.97

21. Robnett TJ, Machtay M, Vines EF, McKenna MG, Algazy KM, McKenna WG. Factors predicting severe radiation pneumonitis in patients receiving definitive chemoradiation for lung cancer. International Journal of Radiation Oncology Biology Physics. 2000;48(1):89-94. doi:10.1016/S0360-3016(00)00648-9

22. Rosenthal M, Cramer D, Bain SH, Denison D, Bush A, Warner JO. Lung function in white children aged 4 to 19 years: II-Single breath analysis and plethysmography. Thorax. 1993;48(8):803-808. 
http://thorax.bmj.com/cgi/doi/10.1136/thx.48.8.803. doi:10.1136/thx.48.8.803

23. Rosenthal M, Bain SH, Cramer D, Helms P, Denison D, Bush A, Warner JO. Lung function in white children aged 4 to 19 years: I-Spirometry. Thorax. 1993;48(8):794-802. http://link.springer.com/10.1007/s00117009-1877-0. doi:10.1136/thx.48.8.794

24. Harness-Brumley CL, Elliott AC, Rosenbluth DB, Raghavan D, Jain R. Gender differences in outcomes of patients with cystic fibrosis. Journal of Women's Health. 2014;23(12):1012-1020. doi:10.1089/jwh.2014.4985

25. Chen Y, Stewart P, Johansen H, McRae L, Taylor G. Sex difference in hospitalization due to asthma in relation to age. Journal of Clinical Epidemiology. 2003;56(2):180-187. doi:10.1016/S0895-4356(02)00593-0

26. Osman M, Tagiyeva N, Wassall HJ, Ninan TK, Devenny AM, McNeill G, Helms PJ, Russell G. Changing trends in sex specific prevalence rates for childhood asthma, eczema, and hay fever. Pediatric Pulmonology. 2007;42(1):60-65. doi:10.1002/ppul.20545

27. Record E, Williamson R, Wasilewski-Masker K, Mertens AC, Meacham LR, Popler J. Analysis of Risk Factors for Abnormal Pulmonary Function in Pediatric Cancer Survivors. Pediatric Blood \& Cancer. 2016;63(7):1264-1271. http://ovidsp.ovid.com/ovidweb.cgi?T $=J S \& P A G E=$ reference $\& D=\operatorname{med} 12 \& N E W S=N \& A N=27002916$. doi:10.1002/pbc. 25969

28. Armenian SH, Landier W, Francisco L, Herrera C, Mills G, Siyahian A, Supab N, Wilson K, Wolfson JA, Horak D, et al. Long-term pulmonary function in survivors of childhood cancer. Journal of Clinical Oncology. 2015;33(14):1592-1600. http://ascopubs.org/doi/10.1200/JCO.2014.59.8318. doi:10.1200/JCO.2014.59.8318

29. Motosue MS, Zhu L, Srivastava K, Stokes DC, Hudson MM, McPherson V, Srinivasan S, Krasin MJ, Green DM, Spunt SL, et al. Pulmonary function after whole lung irradiation in pediatric patients with solid malignancies. Cancer. 2012;118(5):1450-1456. http://doi.wiley.com/10.1002/cncr.26371. doi: $10.1002 /$ cncr. 26371

30. Angela B, Hayley H, Edward A, Robert M. Drug Use among Ontario students: Ontario drug use and health survey 1977-2017. 2017. doi:10.11900/0412.1961.2016.00153

31. Crighton EJ, Feng J, Gershon A, Guan J, To T. A spatial analysis of asthma prevalence in Ontario. Canadian Journal of Public Health. 2012;103(5):384-389.

32. Samuels ML. Large-Dose Bleomycin Therapy and Pulmonary Toxicity. JAMA. 1976;235(11):1117. http://jama.jamanetwork.com/article.aspx?doi=10.1001/jama.1976.03260370025026. doi:10.1001/jama.1976.03260370025026

33. Einhorn L, Krause M, Hornback N, Furnas B. Enhanced pulmonary toxicity with bleomycin and radiotherapy in oat cell lung cancer. Cancer. 1976;37(5):2414-2416. http://link.springer.com/10.1007/978-1-46134349-3_19. doi:10.1002/1097-0142(197605)37:5<2414::AID-CNCR2820370533>3.0.CO;2-J

34. Nygaard K, Smith-Erichsen N, Hatlevoll R, Refsum SB. Pulmonary complications after bleomycin, irradiation and surgery for esophageal cancer. Cancer. 1978;41(1):17-22. doi:10.1002/10970142(197801)41:1<17::AID-CNCR2820410105>3.0.CO;2-Q

\section{Figure Legends}

FIGURE 1. Flow Diagram of Patient Inclusion

FIGURE 2. Trajectory of percent predicted lung function outcomes over time pre and post-treatment by sex (a) Trajectory of Forced Expiratory Volume in 1 Second (FEV1) (b) Trajectory of Diffusion Capacity of the Lungs for Carbon Monoxide Adjusted for Hemoglobin (DLCOadj) (c) Trajectory of Total Lung Capacity (TLC). 
Each point represents the average pulmonary function of one or more individual patients during that onemonth time period. FEV1 was available for $\mathrm{n}=86$ (100\%), DLCOadj for $\mathrm{n}=78(91 \%)$, and TLC for $\mathrm{n}=86$ $(100 \%)$. The $\mathrm{y}$-axis dotted line represents the lower limit of normal for lung function in children. The x-axis dotted line represents the date of treatment completion, or time zero. The blue line represents the trajectory for the overall population.

E-IMAGE 1. Trajectory of percent predicted Forced Expiratory Volume in 1 Second pre and post-treatment in a subset of patients $(\mathrm{n}=65)$ receiving both bleomycin and radiation therapy. $\mathrm{FEV}_{1}$, Forced Expiratory Volume. The y-axis dotted line represents the lower limit of normal for lung function in children. The x-axis dotted line represents the date of treatment completion, or time zero. The blue line represents the trajectory for the overall population.

\section{Hosted file}

Pediatric Pulmonology Tables May 27.docx available at https://authorea.com/users/327309/ articles/454896-trajectory-of-lung-function-change-in-childhood-cancer-survivors-anexploratory-analysis
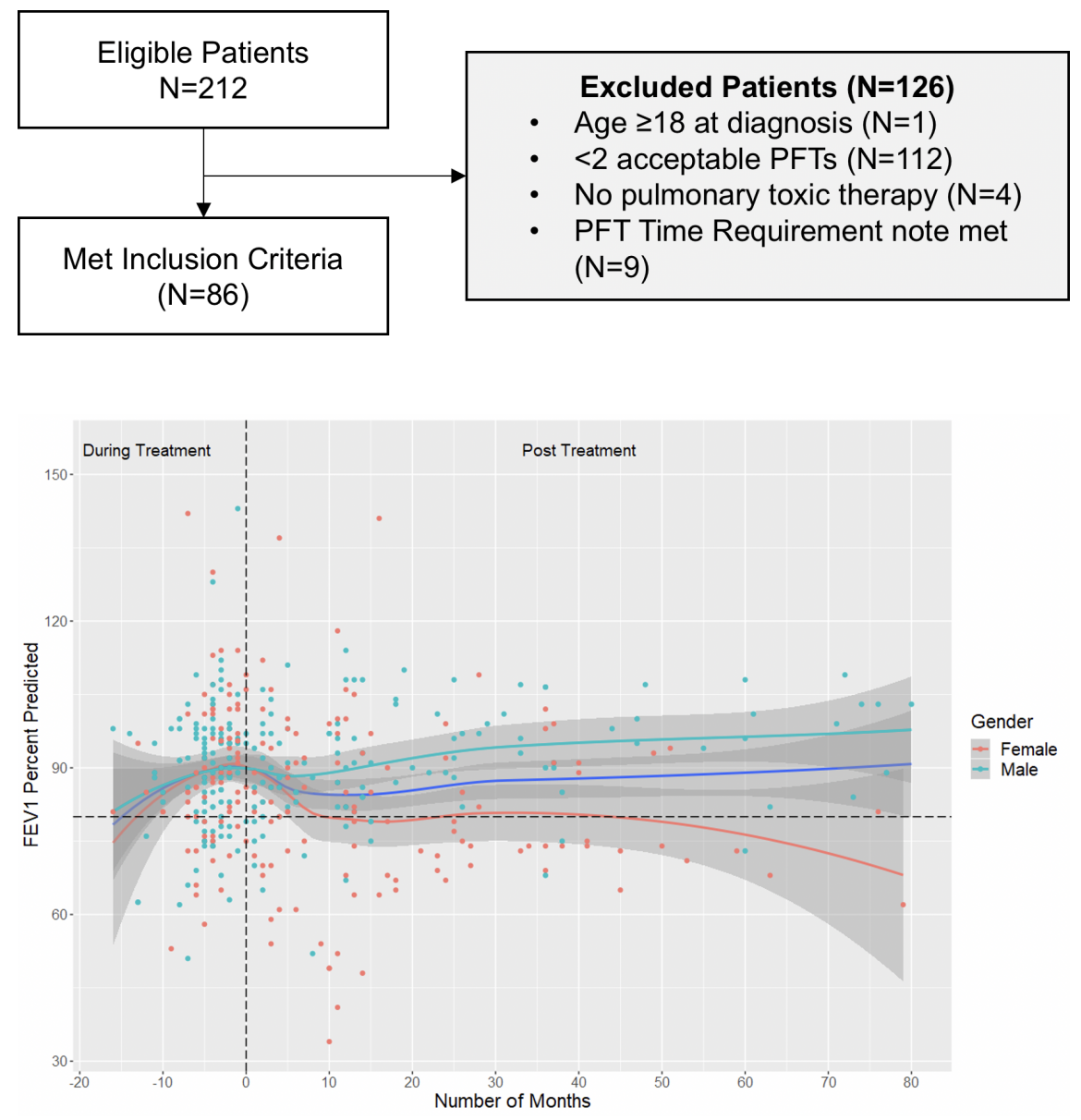

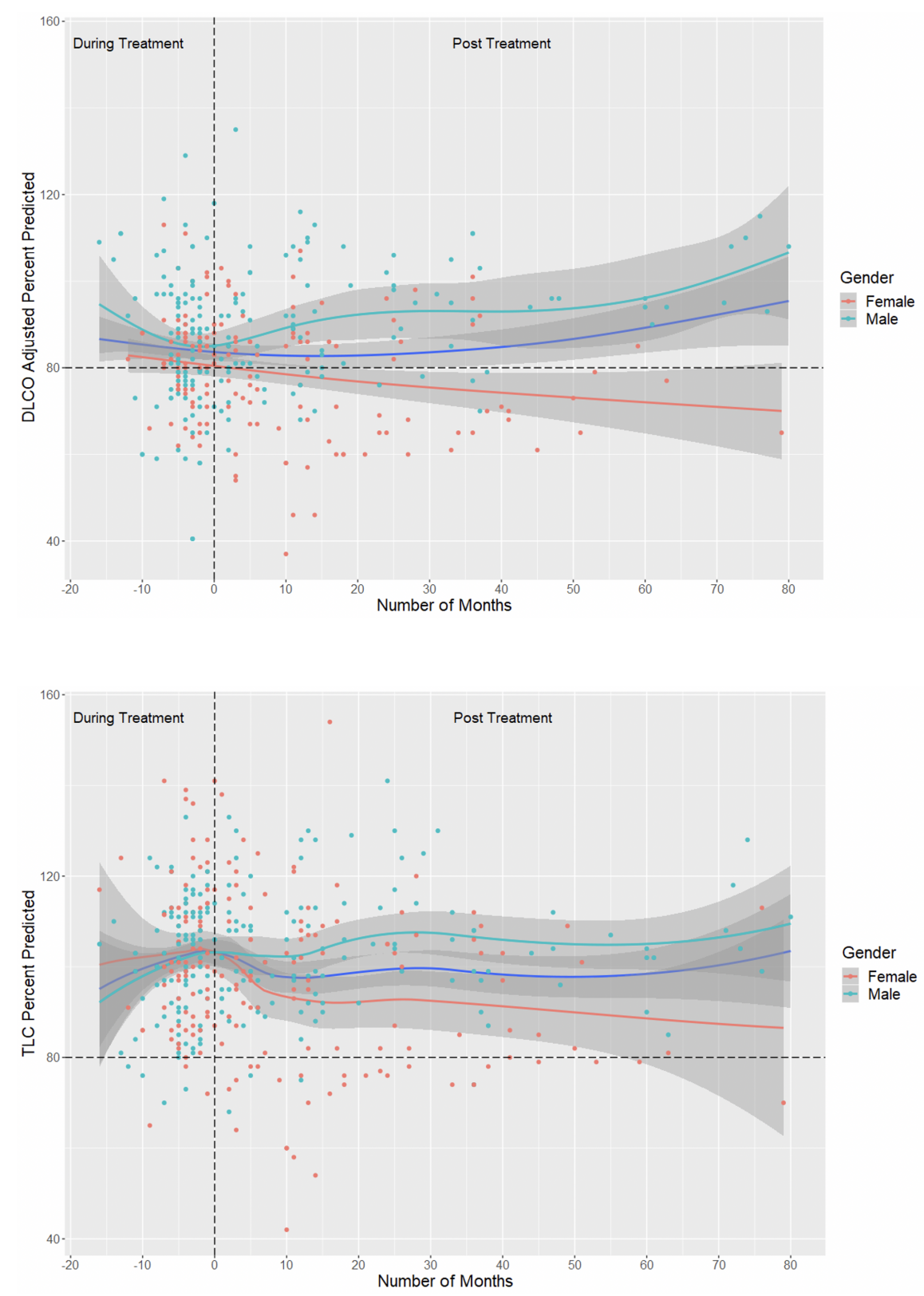

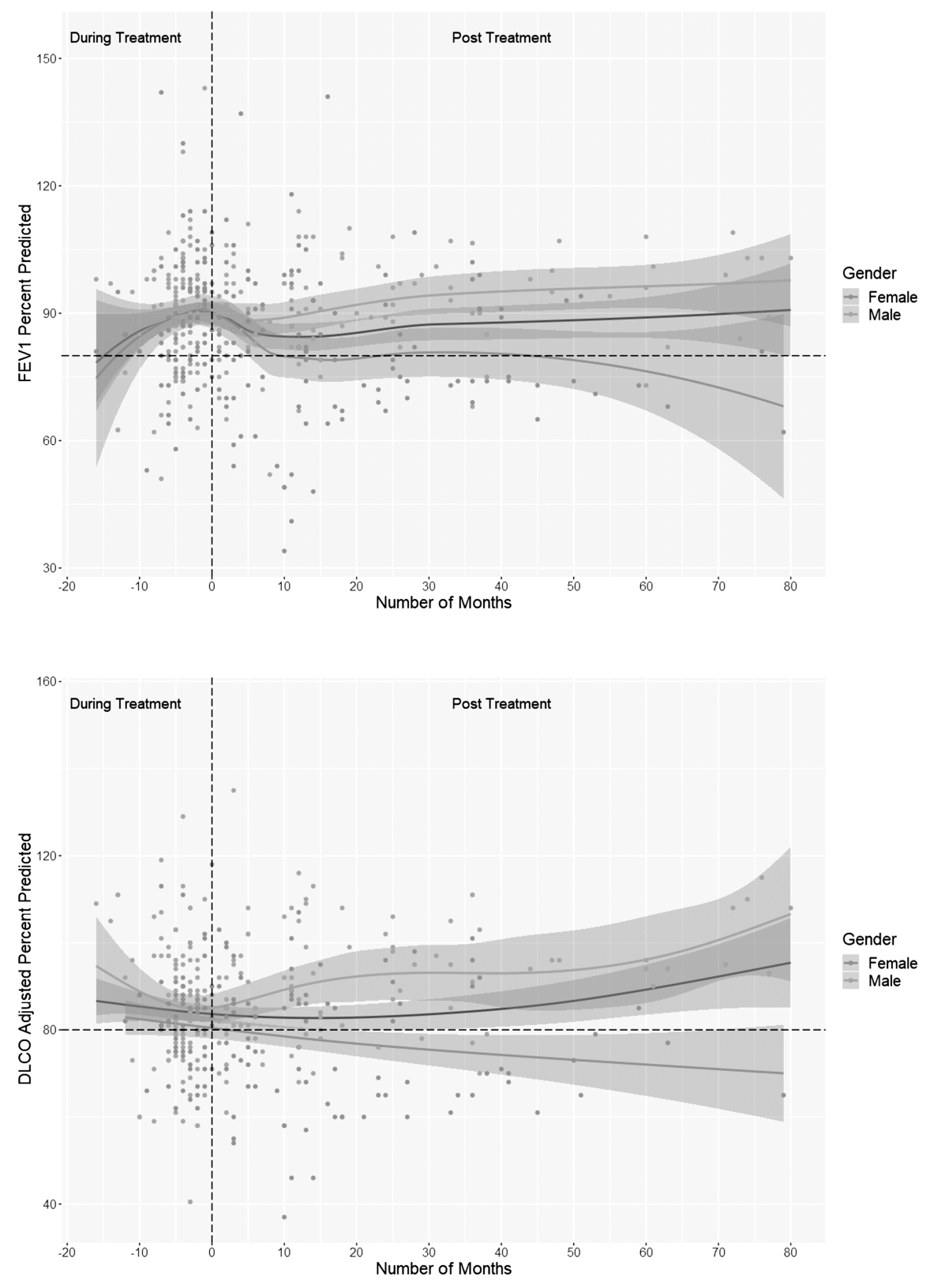


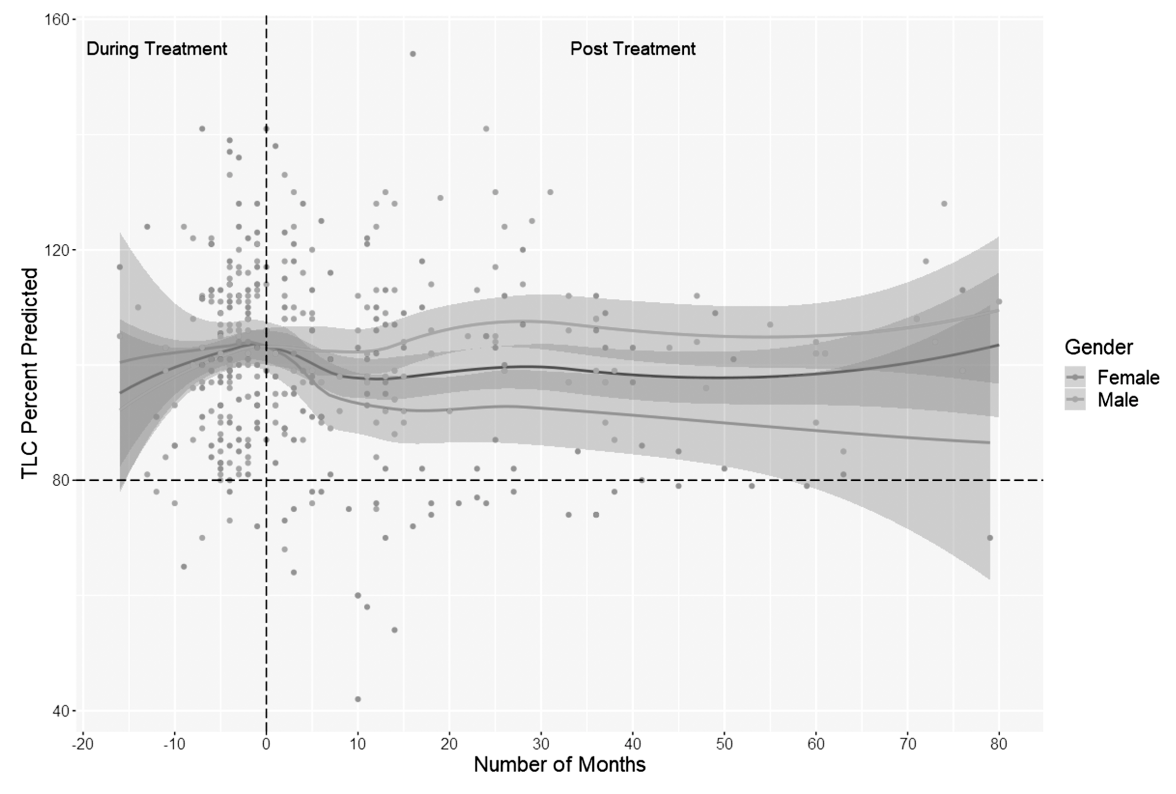

